\title{
Estudio técnico-ambiental de las estructuras principales de fortificación usadas en la minería de carbón de Norte de Santander
}

\author{
A Technical and Environmental Study of Fortified Structures Used \\ in Coal Mining in Norte de Santander
}

Estudo técnico-ambiental das estruturas principais de fortificação
usadas na mineração de carvão do Norte de Santander

Rafael Bolívar-León*

Neyl Richard Triviño-Jaimes**

Javier Mauricio García-Mogollón***

\section{Resumen}

La caracterización de las estructuras de fortificación en la minería de carbón en Norte de Santander (Colombia) y el aporte de este sector a la deforestación han sido poco estudiados. La pérdida de bosques entre 1990 y 2010 en el departamento fue del $26 \%$, y la contribución a ella de la minería de carbón, es de $0,36 \%$. Se determinaron 17 variables geométricas de la estructura, de las cuales sólo dos influyen en el comportamiento mecánico de la estructura. Mediante una simulación estructural y un diseño de experimentos $2^{5}$, se obtuvo un diagrama para la selección del diámetro y el ángulo, el cual garantiza teóricamente un mejor aprovechamiento de la madera. Se podrían aprovechar diámetros de madera tan pequeños como $14 \mathrm{~cm}$. sin detrimento de las propiedades mecánicas del diseño, conllevando un posible menor tiempo de tala de árboles, menor costo de transporte y mayor facilidad de montaje dentro de la mina.

Palabras clave: Estructuras de fortificación, Deforestación, Minería de carbón, Simulación mecánica.

* M. Sc. Metalurgia. Programa Ing. Mecánica. Universidad de Pamplona (Pamplona-Norte de Santander, Colombia). rbolivarl@unipamplona.edu.co ** Ing. Mecánico. Programa Ing. Mecánica. Universidad de Pamplona (Pamplona-Norte de Santander, Colombia). nrichardtj@unipamplona.edu.co

*** M. Sc. Administración. Programa Administración de empresas. Universidad de Pamplona (Pamplona-Norte de Santander, Colombia).xjmg77@yahoo.com 


\section{Abstract}

The mechanic characterization of fortified structures on coal mining at Norte de Santander and the contribution of this sector to the deforestation have been poorly studied. The loss of forests zones between 1990 and 2010 was about $26 \%$, however, the calculation shows that the coal mining sector contribution is only $0,36 \%$. In total, were determined 17 geometric variables on the structures, but only two of them affect significantly their mechanical performance. A mechanical simulation and a $2^{5}$ experimental design were used to obtain a selection diagram of diameter and angle for the structures, which theoretically ensures a better use of wood. It could be used wood diameters as small as $14 \mathrm{~cm}$, without compromising the mechanical properties of the design, leading to a possible shorter logging time, lower transport cost and easier assembly into the mine.

Keywords: Fortification structures, Deforestation, Coal Mining, Mechanical Simulation.

\section{Resumo}

A caracterização das estruturas de fortificação na mineração de carvão no Norte de Santander (Colômbia) e o aporte deste setor à deflorestação têm sido pouco estudados. A perda de bosques entre 1990 e 2010 no Estado foi de $26 \%$, e a contribuição a ela da mineração de carvão, numa análise não conservativa, é de $0,13 \%$. Determinaram-se 17 variáveis geométricas da estrutura, das quais somente duas influem no comportamento mecânico da estrutura. Mediante uma simulação estrutural e um desenho de experimentos $2^{5}$, se obteve um diagrama para a seleção do diâmetro e o ângulo, o qual garante teoricamente um melhor aproveitamento da madeira. Poderiam aproveitarse diâmetros de madeira tão pequenos como $14 \mathrm{~cm}$ sem detrimento das propriedades mecânicas do desenho, implicando um possível menor tempo de corte de árvores, menor custo de transporte e maior facilidade de montagem dentro da mina.

Palavras chave: Estruturas de fortificação, Deflorestação, Mineração de carvão, Simulação mecânica. 


\section{INTRODUCCIÓN}

La minería subterránea de carbón en el departamento de Norte de Santander se realiza de forma tradicional y poco tecnificada; la extracción del mineral se hace en coches jalados por malacates hacia la superficie a través de túneles [1]. La estructura de sostenimiento o entibación de estos túneles es construida en madera; su objetivo es brindar el soporte y la estabilidad mecánica de muros y techos y evitar accidentes por desprendimientos imprevistos de las rocas en el socavón, por insuficiente cohesión [2]. La cantidad de accidentes ocurridos debido a una deficiencia en la estructura es de alrededor del 38\% [3].

Anivel internacional, las tecnologías de entibación más utilizadas en minería subterránea son: autodesplazante, usando pernos de anclaje, con concreto lanzado y con arcos de acero [4-7]. Actualmente, el diseño y la selección de la entibación se realizan en función de análisis geomecánicos en la mina [8-11]; por ello, actualmente las investigaciones se realizan entorno a estas tecnologías.

Las tecnologías de entibación que usan madera se utilizan únicamente en países donde la minería se explota a un muy bajo nivel. La gran mayoría de las unidades mineras de explotación subterránea de carbón en los departamentos de Norte de Santander, Boyacá, Santander y Cundinamarca usan madera para sostenimientos en los socavones. Al ser una tecnología ampliamente usada en este tipo de explotación, y al no encontrarse estudios recientes que determinen la confiabilidad mecánica y el impacto ambiental de dicha estructura, consideramos que este estudio es, entonces, el primer aporte significativo en torno a estos temas.

Legalmente, la madera usada debe ser comprada a bosques reforestados, pero esto no se cumple cabalmente, ya que la minería ilegal hace caso omiso a dicho requerimiento; la minería ilegal en el departamento de Norte de Santander es del orden del 35\% [12-14]. Esta situación, y considerando que el crecimiento de un árbol para alcanzar el tamaño óptimo para corte es de mínimo 4 años, hace prever en un futuro cercano una posible escases de madera estructural para uso en minería (eucalipto, amarillón, alma negra y, algunas veces, pino), y con ello, un incremento de su precio, debido a la alta demanda y al aumento del flete, por la necesidad de usar bosques cada vez más lejanos.

Generalmente, el costo de la madera oscila entre el 8 y el $10 \%$ del total de los costos de explotación, pero podría incrementarse por lo anteriormente planteado. En 2012 y 2013, el sector reportó una producción de 2,396 y 1,88 Mt respectivamente [14].

El sistema de entibación tradicional es conocido como "puerta", que tiene tres partes: un capiz, en la parte de arriba, que sostiene el techo del socavón, actuando como viga, y dos columnas de apoyo, empotradas en el suelo. Los trozos rollizos de madera usados se denominan palancas, independientemente de que sean en vigas o columnas. Generalmente, estas tres partes se unen por apoyo simple, mediante dos muescas en los extremos del capiz y de las columnas, las cuales son elaboradas artesanalmente por los mineros. El ensamble de estas partes se realiza dentro de la mina de forma manual, usando plomadas para dejarlas rectas [15-19]. Se cree que la elaboración manual de las partes de la estructura de entibación, especialmente de la cuña, puede ser un factor que incide negativamente en la confiabilidad de la estructura.

Este trabajo pretende brindar algunos aportes para determinar la deforestación en el departamento de Norte de Santander; para caracterizar mecánicamente, mediante simulación, las entibaciones que se realizan de forma tradicional, usando datos tomados en campo, y para determinar si existe algún parámetro crítico de diseño de las entibaciones, con el propósito de aumentar las condiciones de seguridad o incrementar la vida útil de éstas mediante una modificación en el diseño. Además, está inmerso en una investigación que pretende desarrollar materiales alternos para dar una posibilidad al industrial de reemplazar el uso de la madera [19], bajo el concepto de ingeniería inversa [20-22].

El artículo se compone de dos partes principales. La primera es una determinación de la deforestación de Norte de Santander, con base en datos suministrados por el Instituto de Hidrología, Meteorología y Estudios Ambientales de Colombia (IDEAM); también se realiza un cálculo conservativo de la contribución de la minería a la deforestación. En la segunda parte se determina la confiabilidad mecánica de la 
entibación, con base en los parámetros identificados de su estructura; dicha determinación se realiza por un diseño de experimentos estadístico y simulaciones mecánicas; los parámetros de diseño de la estructura, y sus magnitudes fueron determinados por un análisis de las diferentes geometrías encontradas en algunas minas de Norte de Santander.

\section{Materiales Y MÉTOdoS}

\section{A. Determinación de la deforestación}

Usando los datos obtenidos a través de la interpretación automatizada de imágenes satelitales, con una resolución espacial de 30 m, del IDEAM, se comparó la deforestación entre 1990 y 2010 , y se realizó una proyección al año 2015 [23].

\section{B. Determinación de la geometría de la entibación}

Para caracterizar el sistema de entibación se identificaron los parámetros involucrados en el diseño de las puertas de minería [19]. Los valores de estos parámetros se recolectaron por medio de visitas a diferentes minas de carbón de la región, donde se determinaron las medidas de las disposiciones geométricas, tipos de maderas y procedimientos de construcción de la estructura, en especial la cuña.

Con las formas encontradas de las puertas se realizó un análisis geométrico, tomando como constante el valor del área de la puerta, de $3 \mathrm{~m}^{2}$, y su altura, de 1,8 $\mathrm{m}$, y se fijaron los siguientes límites de frontera: 1) el valor del diámetro de las columnas y del capiz sería el mismo, 2) los valores de las longitudes del capiz y la columna se establecerán en la parte interna de la puerta para garantizar el área proporcionada por ella, 3) el tamaño de la muesca será igual al valor del diámetro, 4) las áreas de las cuñas estarán plenamente en contacto.

El propósito del análisis fue determinar las longitudes y los ángulos independientes en el diseño, que se llamarán desde ahora variables independientes.

\section{Diseño experimental factorial $2^{5}$}

Para determinar la influencia de las variables independientes en el comportamiento mecánico de la estructura se realizó un diseño de experimentos $2^{5}$, donde se usaron como factores las cinco variables independientes encontradas en el análisis geométrico, y como niveles o valores del factor, los máximos y mínimos encontrados en los datos recolectados en campo. El diseño $2^{5}$ arrojó 36 configuraciones diferentes, con cuatro de ellas tomando el valor del punto medio de los niveles de las variables; estas configuraciones se simularon mecánicamente. El resultado del diseño (variable respuesta esfuerzo de Von Mises) se analizó usando el software DesignExpert 8.0.7.1 trial [24-25].

\section{Simulación mecánica}

Para cada configuración determinada por el diseño factorial $2^{5}$, se realizó una simulación mecánica en el software SolidWorks 2010, usando la herramienta SolidWorks Simulation, y obteniendo como variable respuesta el esfuerzo de Von Mises.

Se realizó el modelado de las piezas que conforman la puerta (las palancas y el capiz), definiendo las relaciones de posición y condiciones de contacto entre las superficies que conforman la muesca con relaciones de coincidencia entre caras de ensamble. Se definieron las sujeciones del modelo, como una geometría fija en la base de las palancas. Se realizó el mallado con parámetros estándar de 64,7 x 3,2 mm. Finalmente, se seleccionó el eucalipto seco al aire, al $12 \%$, como el material a simular, debido a su mayor uso en la fabricación de puertas para la minería en la región.

Para determinar el valor de la carga por aplicar se realizó un procedimiento iterativo con respecto al valor teórico de ruptura del eucalipto $(1,089 \mathrm{MPa})$, teniendo en cuenta los valores máximos y mínimos de las variables independientes anotadas anteriormente. Cuando el error relativo entre el módulo de ruptura y el valor del esfuerzo de Von Mises fue menor al 0,1\%, se supuso que era la carga máxima antes de la falla con las que se simularían todas las configuraciones. 


\section{RESULTADOS Y DISCUSIÓN}

\section{A. Deforestación en Norte de Santander en el período 1990-2010}

La Fig. 1, elaborada con base en el Proyecto "Monitoreo de la deforestación mediante el procesamiento digital de imágenes", del SIAC, permite comparar la deforestación entre los años 1990 y 2010 [23], así como hacer una proyección al año 2015.

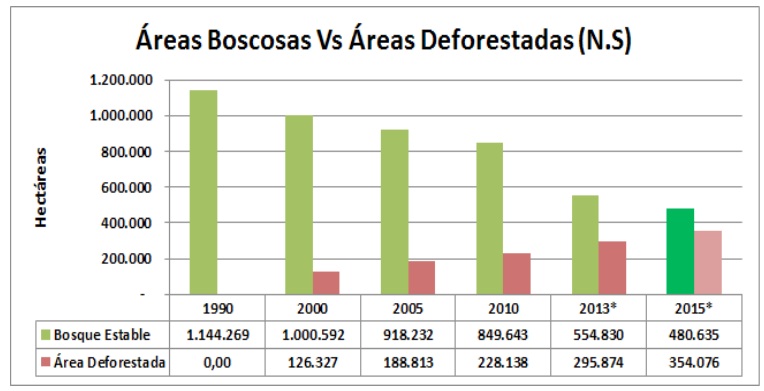

* Proyecciones estimadas para los años 2013 y 2015

Fig. 1. Áreas boscosas en Norte de Santander 1990 con proyección a 2015

Como se ve en la Fig. 1, en el año 2010 se identificó una superficie de cobertura boscosa total para el departamento de 849643 ha, con una pérdida de 294 626 ha entre 1990 y 2010 , que representa el $36 \%$ de pérdida total de zona boscosa; de este porcentaje, el $30 \%$ se debió a deforestación, y el restante, a otros factores como minería, ganadería y urbanismo [23]. Proyectando los datos obtenidos mediante una relación lineal, al 2015, se puede suponer que la superficie boscosa se reducirá a 480635 ha, con una pérdida de 663637 ha en comparación con 1990, es decir, $58 \%$ del área de bosque estable habrá sido pérdida en comparación con la registrada en 1990; de este porcentaje, $31 \%$ sería de deforestación y el restante $27 \%$ a otros factores.

Se estima, según encuestas realizadas a diferentes unidades mineras, que actualmente se usa 0,5 palancas por tonelada de carbón extraído, una puerta de entibación está conformada por 3 palancas y se consume aproximadamente 0,7 árboles por puerta. Las puertas, según las condiciones de la mina y el tratamiento previo de la madera, exigen un recambio después de 6 meses o un año. De aquí se puede inferir que se usan 0,116 árboles por tonelada de carbón anualmente, sin considerar el recambio de éstas; es decir, teniendo en cuenta la producción de toneladas para 2012 y 2013, anotadas arriba, se calcula que se usaron aproximadamente 279533 y 219933 árboles, respectivamente. Un cultivo de eucalipto produce, en promedio, 1350 árboles por hectárea, lo que arrojaría que en estos dos años se talaron en el departamento, con fines de minería, 207 y 162 ha de bosque maderable, respectivamente.

Tomando el dato superior, es decir, las 207 ha por año, y calculando a 20 años, serían 4140 ha consumidas por minería de carbón, es decir, que la minería podría llegar a ser responsable del $0,36 \%$ de la deforestación entre 1990-2010, sin tener en cuenta la minería ilegal. El restante valor se podría deber a otras causas habituales de deforestación como la colonización no planeada, la agricultura y la ganadería extensiva. Es importante aclarar que la proyección se realiza con la cantidad de toneladas producidas en 2012, año que registró la máxima producción desde 1990. Sin embargo, a pesar que es un porcentaje insignificante en comparación con la deforestación total de Norte de Santander, si esta se hiciera únicamente con las especies maderables usadas en la minería, creemos sería bastante alta. Esta hipótesis, se fundamenta porque algunos mineros ya vienen manifestando escases de este insumo. Desafortunadamente el inventario forestal departamental, no cuantifica las hectáreas de bosques por especie.

\section{B. Análisis del diseño, simulación y diseño experimental}

Se identificaron 17 variables en la estructura: 7 ángulos, 9 longitudes y 1 diámetro (Fig. 2). El análisis geométrico arrojó 5 variables independientes: la longitud de la cuña $\left(L_{1}\right)$, la inclinación de la palanca $\left(\theta_{2}\right)$, el diámetro de la madera (D) y los ángulos de la cuña $\left(\alpha_{2}\right)$ y $\left(\alpha_{4}\right)$. 


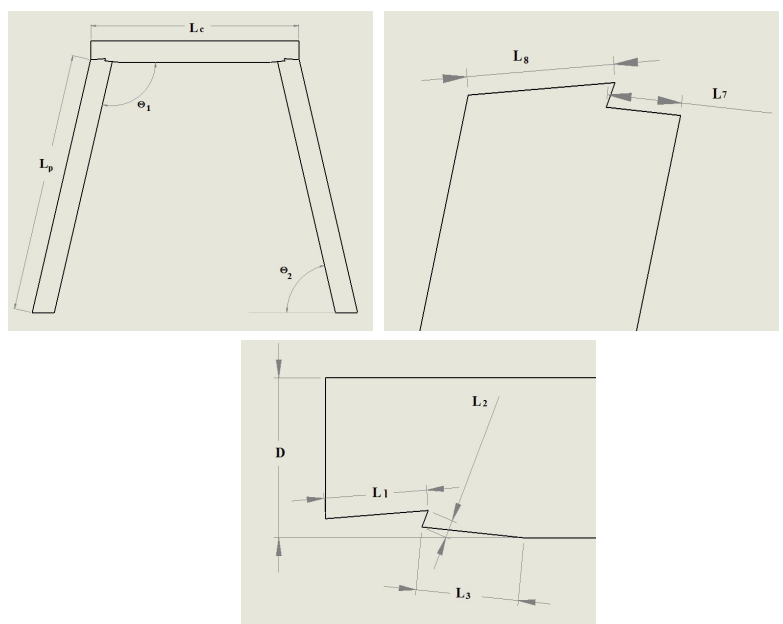

Fig. 2. Variables de las estructuras de las puertas de minería

Los niveles máximos y mínimos definidos de las 5 variables encontradas se pueden apreciar en la Tabla 1.

\section{TABLA 1}

MíNIMOS Y MÁXIMOS DE LAS VARIABLES

INDEPENDIENTES EN LAS PUERTAS DE MINERÍA

\begin{tabular}{|l|l|l|}
\hline \multicolumn{1}{|c|}{ Variable } & \multicolumn{1}{c|}{ Mínimo } & \multicolumn{1}{c|}{ Máximo } \\
\hline$L_{1}=$ longitud de la cuña & $5,5 \mathrm{~cm}$ & $8 \mathrm{~cm}$ \\
\hline$\theta_{2}=$ inclinación de la palanca & $74^{\circ}$ & $84^{\circ}$ \\
\hline$D=$ diámetro & $14,6 \mathrm{~cm}$ & $18 \mathrm{~cm}$ \\
\hline$\alpha_{2}=$ ángulo en la cuña & $66^{\circ}$ & $90^{\circ}$ \\
\hline$\alpha_{24}=$ ángulo en la cuña & $67^{\circ}$ & $90^{\circ}$ \\
\hline
\end{tabular}

La simulación mostró que los valores de las cargas de falla máxima y mínima que se obtuvieron fueron $585,4 \mathrm{~kg}$ y $357,4 \mathrm{~kg}$, respectivamente, y que existían tres puntos críticos en la estructura, los cuales se ubican en la parte superior del centro del capiz (punto 1), en la parte inferior del centro del capiz (punto 2) y en el punto adyacente a la cuña en la palanca, como se muestra en la Fig. 3.

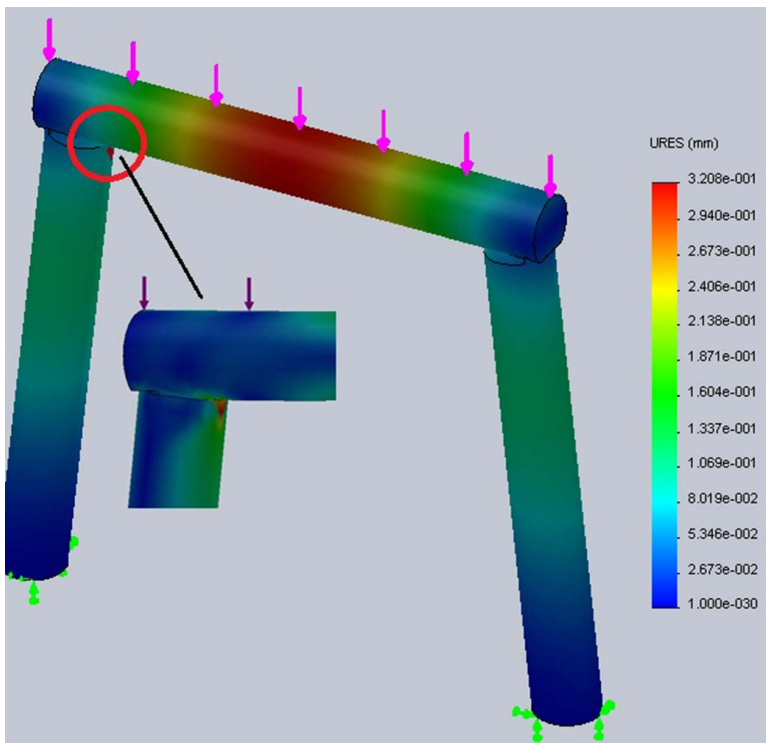

Fig. 3. Nivel del esfuerzo de Von Mises según las variables influyentes y puntos críticos en la estructura $(1,2,3)$

Se alimentó la matriz del diseño de experimentos $2^{5}$ con los valores de los esfuerzos de Von Mises (la variable respuesta) obtenidos en cada una de las simulaciones, y mediante el análisis estadístico respectivo (ANOVA) se determinó el comportamiento de las 5 variables y mostró validez estadística.

Se encontró que únicamente dos variables, el diámetro (A) y el ángulo de inclinación de la palanca el ángulo $\theta_{2}(\mathrm{~B})$, y su interacción $(\mathrm{AB})$ (Tabla 1) eran influentes en el valor del esfuerzo de Von Mises. La contribución de estas variables es mostrada en la Tabla 3 , donde se aprecia que el diámetro es el factor más sensible, con una contribución al esfuerzo de Von Mises de $76 \%$ para los puntos 1 y 2 de la estructura; pero para el punto 3 su contribución es casi la mitad, de $45 \%$, potenciando la contribución del ángulo $\left(\theta_{2}\right)$ y de la interacción del diámetro con el ángulo (Diámetro* $\theta_{2}$ ). 


\section{TABLA 3}

CONTRIBUCIÓN DE LOS FACTORES EN EL COMPORTAMIENTO MECÁNICO EN LOS PUNTOS CRÍTICOS

\begin{tabular}{|l|l|c|c|}
\hline \multirow{2}{*}{ Puntos } & \multirow{2}{*}{ Factor } & \multicolumn{2}{|c|}{ \% Contribución } \\
\cline { 3 - 4 } & & C. Máxima & C. Mínima \\
\hline \multirow{4}{*}{ Punto 1 } & Diámetro & 77,43 & 77,47 \\
\cline { 2 - 4 } & Teta 2 & 9,12 & 9,06 \\
\cline { 2 - 4 } & Interacción* & 7,87 & 7,87 \\
\hline \multirow{4}{*}{ Punto 2 } & Diámetro & 74,92 & 74,94 \\
\cline { 2 - 4 } & Teta 2 & 11,43 & 11,35 \\
\cline { 2 - 4 } & Interacción* & 8,84 & 8,91 \\
\hline \multirow{4}{*}{ Punto 3 } & Diámetro & 45,02 & 45,09 \\
\cline { 2 - 4 } & Teta 2 & 30,55 & 30,51 \\
\cline { 2 - 4 } & Interacción* & 20,10 & 19,93 \\
\hline \multirow{3}{*}{ * Interacción de los factores Diámetro por Teta 2 } \\
\hline
\end{tabular}

Se calcularon las ecuaciones del modelo estadístico teniendo en cuenta las variables influyentes, obteniendo una ecuación potencial cúbica para cada punto, que fue graficada como se muestran en las gráficas de la Fig. 4. Los números dentro del contorno representan el esfuerzo de Von Mises; el eje X representa el diámetro de la palanca, y el eje Y, el ángulo .

Como se ve en la Fig. 4, los menores esfuerzos de Von Mises se obtienen a mayores diámetros, por ejemplo, a un diámetro de $16 \mathrm{~cm}$ y un ángulo de $80^{\circ}$ en los punto 1 y 2 se inducirá un esfuerzo de 1,05 $\mathrm{MPa}$ (arriba), pero en el punto 3 (abajo) se ejercerá uno de 1,16 MPa.

El color de azul a rojo indica el incremento en el esfuerzo interno del material hasta alcanzar falla, es decir, el diseño nunca debe alcanzar el color rojo. Obsérvese que de las dos gráficas, la del punto 1 y 2 son las que menores esfuerzos de Von Mises alcanzan antes de falla, lo que conduce a que éstas son las que deben usarse para seleccionar los parámetros más convenientes.
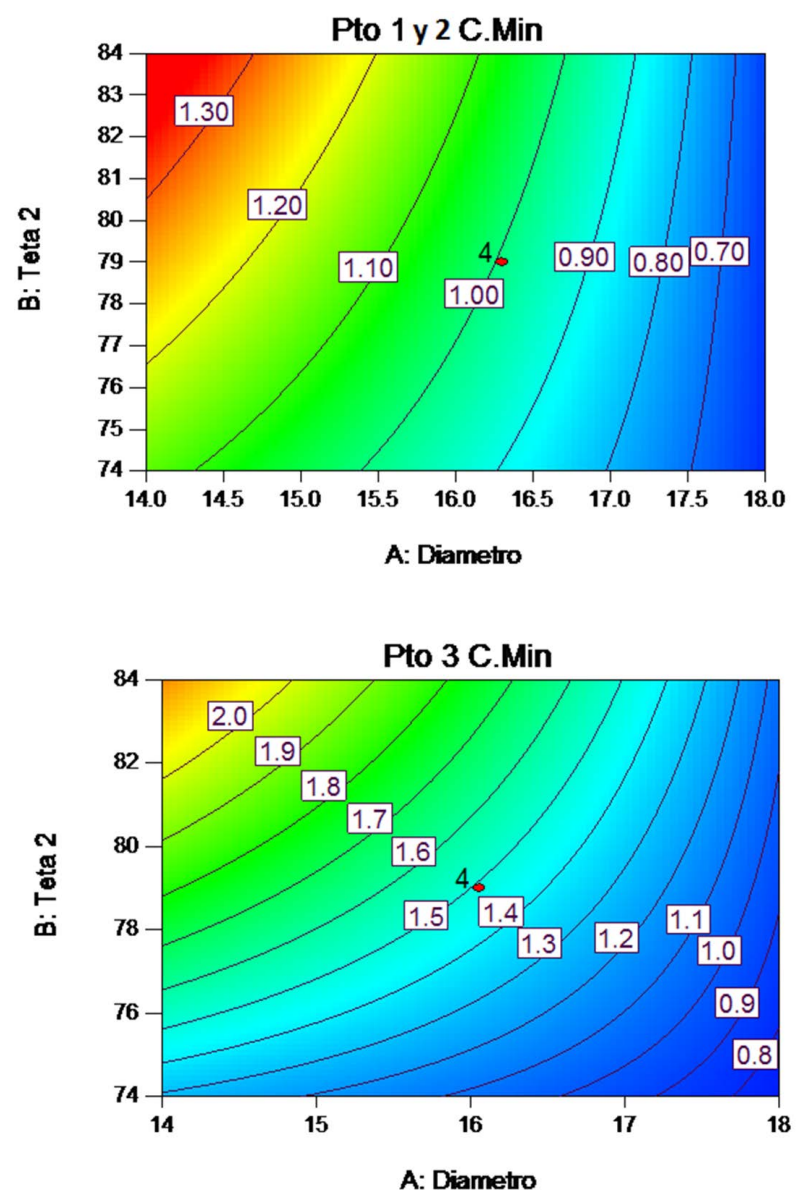

Fig. 4. Gráficos de nivel del esfuerzo de Von Mises según las variables influyentes en los puntos críticos

Esta figura puede ser usada en campo para seleccionar el diámetro de la madera y un ángulo específico durante la instalación de la puerta donde sea posible, según la formación geológica interna de la mina, es decir, si se tienen palancas de diámetros pequeños, se deberían buscar unos ángulos más agudos para asegurar un menor esfuerzo en el punto 3.

Este hecho contribuye directamente a que el minero podría usar para puertas maderas con espesores tan bajos como de $14 \mathrm{~cm}$, diámetros que comúnmente son despreciados; con ello, incluso, podría hacerse el talado del árbol más rápido. La gráfica de superficie respuesta (Fig. 5) del punto 1 y 2 también puede usarse para la selección en campo; sin embargo, su importancia es desde el punto de vista estadístico, y muestra que no existen máximos o mínimos en superficie, o sea que el comportamiento de los modelos es lineal. 


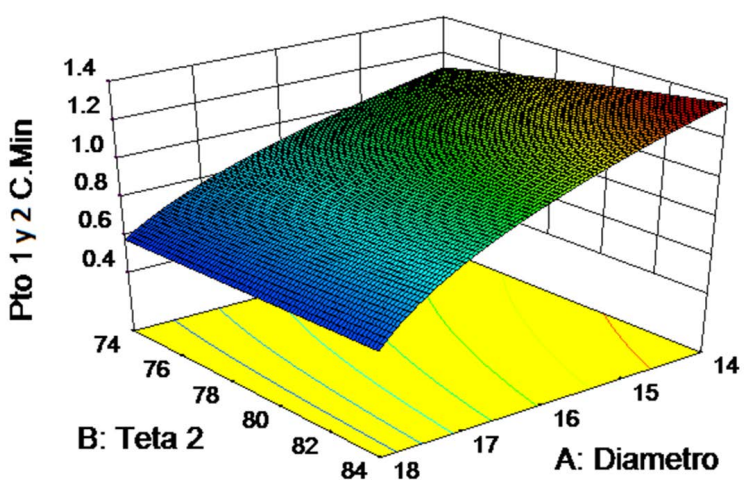

Fig. 5. Superficie respuesta en función de los parámetros influyentes para el punto 1 y 2

\section{Conclusiones}

Las mayores áreas de coberturas boscosas significativamente afectadas se ubican en la región del Catatumbo, en el centro y en el sur-oriente del departamento. La superficie de bosque en el 2010 fue de 849643 ha, que representó una disminución de $26 \%$ del área contabilizada en el año 1990, de la cual el $20 \%$ se estima que es causada por deforestación.

Se proyecta que la superficie de bosque para el 2015 será de unas 480635 ha, que significaría una disminución de 58\% del área contabilizada en el año 1990, de los cuales, el 31\% se estima que es causada por deforestación. El alto consumo de madera ha trasformado significativamente los ecosistemas forestales de Norte de Santander en los períodos 1990-2000 y 2005-2010, causando la desaparición y degradación de los bosques, lo cual indica que no ha sido sostenible su explotación.

Tomando la mayor producción del departamento durante los años 1990 a 2010, y suponiendo que ésta fue realizada durante todos el período de tiempo, se puede concluir que la minería legal, aportaría a la deforestación apenas en el $0,36 \%$ del total calculado entre los años 1990 a 2010 ; el otro $99,64 \%$ de deforestación se debería a otras causas, como la colonización no planeada, la agricultura y la ganadería extensiva.

La deforestación se ha calculado de forma general, teniendo en cuenta toda la zona boscosa del departamento, y no de forma específica sobre las especies maderables que usa la minería de carbón (amarillón, alma negra, eucalipto y pino). Por ello la participación de este sector es de un insignificante $0,36 \%$. Sin embargo, los mineros de la región manifiestan que existe una situación actual en la que este tipo de especies ha empezado a escasear, lo que determina, que la deforestación de estas especies debe ser bastante alta. Esta situación implicará un aumento en el costo de producción por tonelada de carbón debido al incremento del costo de dicho insumo y del coste del flete, poniendo en peligro la continuidad de las unidades mineras más tradicionales.

De todos los factores considerados en el diseño, es decir, el diámetro, el ángulo de inclinación de la palanca $\left(\theta_{2}\right)$, el diámetro (D), la longitud en la cuña $\left(\mathrm{L}_{1}\right)$ y los ángulos en la cuña $\left(\alpha_{2}, \alpha_{4}\right)$, se determinó estadísticamente que únicamente el diámetro y el ángulo, y su interacción, influyen en el comportamiento mecánico de la estructura. Es decir, la práctica tradicional de hacer las cuñas en campo sin ninguna geometría específica es válida mecánicamente, teniendo en cuenta las consideraciones planteadas anteriormente.

Es posible seleccionar las palancas de madera y construir la estructura de la puerta con diámetros tan pequeños como $14 \mathrm{~cm}$. en sitios donde pueda acondicionarse el ángulo, aprovechando entonces palancas con diámetros que muchas veces no son usadas, y asegurando un comportamiento mecánico confiable. Con ello podría talarse más rápido el árbol, y se haría más fácil el montaje dentro de la mina, al ser mucho más livianas las estructuras.

Es necesario realizar una comprobación a nivel laboratorio y en campo del comportamiento mecánico de la estructura, usando los parámetros de diseño encontrados en la simulación para poder asegurar la confiabilidad en condiciones reales. Después de la comprobación de la confiabilidad de estos parámetros se podrán realizar entrenamientos a los mineros para que puedan hacer el montaje de las estructuras con la disposición geométrica y el dimensionamiento propuesto por esta investigación. 


\section{REFERENCIAS}

[1] República de Colombia, Ministerio de Minas, ABC Minero, 2012.

[2] República de Colombia, Ministerio de Minas, Censo Minero Departamental Colombiano. 2012.

[3] M. C. García Botero, Informe de gestión 2012. Bogotá: Grupo Edición Agencia Nacional Minera, 2012.

[4] R. J. Ferreras, C. G. Nicieza, A. M. Díaz, A. E. Vigil, and M. I. Álvarez, "Measurement and analysis of the roof pressure on hydraulic props in longwall",Int. J. Coal Geol., vol. 75, no. 1, pp. 49-62, Jun. 2008.

[5] X. N. Tan and H. Qin, "Bolt support mechanism based on elastic theory",Int. J. Min. Sci. Technol., vol. 23, no. 4, pp. 469-474, 2013.

[6] C. Banton, M. S. Diederichs, D. J. Hutchinson, and S. Espley, "Mechanisms of Shotcrete Roof Support", in Taylor \& F., E. S. Bernard, Tunnelling and Underground Space Technology, pp. 39-46 London, 2004.

[7] H. S. Mitri and F. P. Hassani, "Structural characteristics of coal mine steel arch supports," Int. J. Rock Mech. Min. Sci. Geomech. Abstr., vol. 27, no. 2, pp. 121-127, Apr. 1990.

[8] C.A. Oztur, "Support design of underground openings in an asphaltite mine". Tunnelling and Underground Space Technology, vol. 38, pp. 288-305, Sep. 2013.

[9] P. Forrest, E. Schumachera, "Modeling the pipe umbrella roof support system in a Western US underground coal mine". International Journal of Rock Mechanics and Mining Sciences, Vol. 60, pp. 114-124. June 2013.

[10] Y. Kanga, Q. Liua, G. Gongc, H.Wangd, "Application of a combined support system to the weak floor reinforcement in deep underground coal mine",International Journal of Rock Mechanics and Mining Sciences, vol. 71, pp. 143-150, 2014.

[11] E. Ghasemia, M. Ataeia, K. Shahriarb, F. Sereshkia, S. E. Jalalia, A. Ramazanzadeha,"Assessment of roof fall risk during retreat mining in room and pillar coal mines", International Journal of Rock Mechanics and Mining Sciencesm, Vol. 54, pp. 80-89,Sep.2012.

[12] Fedesarrollo, Pequeña y mediana minería de carbón del interior del país: alternativa de financiación y comercialización a partir de la conformación de las alianzas estratégicas, 2011. Disponible en: http://www.fedesarrollo.org.co/ wp-content/uploads/2011/08/Peque\%C3\%B1ay-median a-mine r \% C $3 \%$ A D a - d e carb $\% \mathrm{C} 3 \% \mathrm{~B} 3 n$-del-interior-del-pa $\% \mathrm{C} 3 \%$ ADsInforme-final-19-de-diciembre.pdf.

[13] L.A. Arévalo, Manual para la identificación de maderas que se comercializan en el departamento de Tolima, ISBN: 958-33-88491, Ibagué, 2005.

[14] Sistema Nacional de Información MineroColombiano, SIMCO, Producción $y$ exportaciones de carbón relacionada con producción de carbón por departamentos, 2013. Disponible en: www.simco.gov.co.

[15] F. Ortiz de Urbina, J. Herrera, Curso de Laboreo de Minas, Madrid, 2002.

[16] República de Colombia, Ministerio de Minas, Métodos de explotación minera. Dirección General de Minas, 2010.

[17] H.J. Vallares, Cálculo y diseño de entibaciones para excavaciones en profundidad. Tesis Ingeniero constructor. Valdivia Chile. Universidad Austral de Chile. Facultad de Ciencias de Ingeniería. Escuela de Construcción Civil, 2007.

[18] Departamento de Seguridad Minera, Guía metodológica de seguridad para sistemas de fortificación y acuñadura, Chile, 2010.

[19] L.M. Bermúdez, Caracterización mecánica del sistema de entibación de algunas minas tradicionales de carbón en Norte de Santander. Tesis Ingeniería Mecánica, Universidad de Pamplona, Colombia, 2013.

[20] N. Rozo, G. D. Marín, J. D. Giraldo, D. A. Cardona, J. M. Meza. "Ingeniería inversa de un arco recurvo compuesto". VII Congreso internacional de Materiales. Medellín, 2013. 
[21] M.F. Ashby, Materials Selection in Mechanical Design, $4^{\circ}$ Edition. Butterworth-Heineman, Oxford, 2010.

[22] R.B. Barbero, "The recovery of design intent in reverse engineering problems". Computers \& Industrial Engineering. Vol. 56, No. 4, pp. 12651275. May 2009.

[23] República de Colombia, Instituto de Hidrología y Estudios Ambientales, IDEAM, Monitoreo de la deforestación mediante el procesamiento digital de imágenes, 2011. Disponible en: http:// bitly.com/1vIB5jR.

[24] P. Box and S. Hunter,"Diseño factorial a dos niveles". En: Estadística para investigadores, Cap. 10, Colombia: Ed. Reverté, pp. 317, 2002.

[25] State Easy, Manual General One-Factor Tutorial Design-Expert 8. Disponible en: http:// www.qualitycoach.net/land/design-expert/ tutorials/DX8-02A-Gen1Factor-P1.pdf. 